\title{
Possible mechanism of inhibition of virus infectivity with nanoparticles
}

\author{
O.L. Khylko, N.M. Rusinchuk \\ Institute of High Technologies, Taras Shevchenko National University of Kyiv \\ 4-g, prosp. Glushkova, 03022 Kyiv, Ukraine \\ Tel.+380(44)521-35-66; e-mail: o.khylko@gmail.com;n.m.rusinchuk@gmail.com
}

\begin{abstract}
The possible mechanism of inhibition of virus infectivity with nanoparticles has been considered both theoretically and experimentally. It has been supposed that inhibition is caused by action of the nanoparticles on specific molecules at the virus surface. A nanoparticle located close to the virus leads to the drastic increase in the local field intensity at this surface. It, in turn, leads to deformation (or destruction) of molecules that are responsible for virus absorption into the cell. When majority of these molecules are deformed, the virus loses its ability to penetrate into the cell and to cause a decease. This mechanism has been studied theoretically by applying the methods of nanophysics and experimental studying the absorption spectra of biological samples.
\end{abstract}

Keywords: antiviral therapy, virus, nanoparticles, local field, effective susceptibility, absorption spectrum.

Manuscript received 15.01.16; revised version received 19.04.16; accepted for publication 08.06.16; published online 06.07.16.

\section{Introduction}

Viruses have been a relevant object of research for a long time. At first, scientists tried to develop different methods of dealing with viruses. Afterwards, viruses began to be used for various purposes, e.g., for drug delivery $[1,2]$ or for fighting bacteria $[3,4]$ and even other viruses [5].

Nowadays, the physical methods are applied more and more actively in virology. Nanophysics has also opened new opportunities for antiviral therapy. Rapid development of nanotechnologies led to appearance of a great deal of new antiviral techniques and medicines that were successfully tested both experimentally and clinically.
Recently, a lot of results concerning the antiviral properties of nanoparticle preparations are reported. For instance, the antiviral activity of titanium dioxide nanoparticles about $4-5 \mathrm{~nm}$ in size against the H3N2 influenza virus was reported [6]. Electron microscopy studies showed that the nanoparticles stuck to the external virus shell already in $15 \mathrm{~min}$ and induced its local destructions, which resulted, in turn, in viruses loosing their infectious ability. The authors in $[7,8]$ devote their studies to antiviral action of pure silver nanoparticles preparations against $\mathrm{HIV}-1$, and the authors of [9] carried out the same experiments for the Herpes Simplex Virus 2. The pure nanoparticles of other type samples have demonstrated their antiviral effect in a lot of experiments that was carried out by different 
research teams [7-11]. Since the antiviral activity is observed for a large variety of nanoparticles, it is possible to suppose that a certain physical mechanism not connected with chemical interaction governs all that. The possible mechanisms that could describe the antiviral effect of the nanoparticles were suggested and described in detail by the authors of [10].

In order to study the possible mechanism of interaction between the virus and nanoparticle, the effect of the nanoparticle presence on the local field near the virus particle needs to be studied and obtained theoretical results need to be compared with experimental results of other works. Furthermore, as absorption spectra of the sample depends on its content and structure, the optical studies of the samples can give an information on the mechanism of the inhibition of the virus infectivity.

\section{The local field in the presence of a nanoparticle}

In order to calculate the local field formed in the course of interaction between the nanoparticle and surface, the following model (Fig. 1) was chosen two nanoobjects with spherical shapes that are located at the distance $d$ between them. The first one corresponds to metal or semiconductor nanoparticle with the radius $r_{1}$ and the second one corresponds to the virus with the radius $r_{2}$ and some shell thickness $s$. The particles are placed along $\mathrm{X}$-axis, and wave vector of external field rotates in XOZ plane.

It should be noted that from the physical viewpoint most of viruses can be considered as nanoparticles, since their linear dimensions are close to $100 \mathrm{~nm}$ [12-14]. As it is well known, viruses have various shapes, so the spherical shape was chosen as the simplest one to be calculated, and since the shape of such widespread viruses as Human Immunodeficiency Virus (HIV) and Influenza Virus is the same. The virus is not a homogeneous system and cannot be described as a homogeneous nanoparticle. As the virus consists of at least two parts, which are the core with DNA or RNA and the shell (or capsid) with proteins protecting its genetic information, it was decided to apply a model of a nanoparticle with a shell.

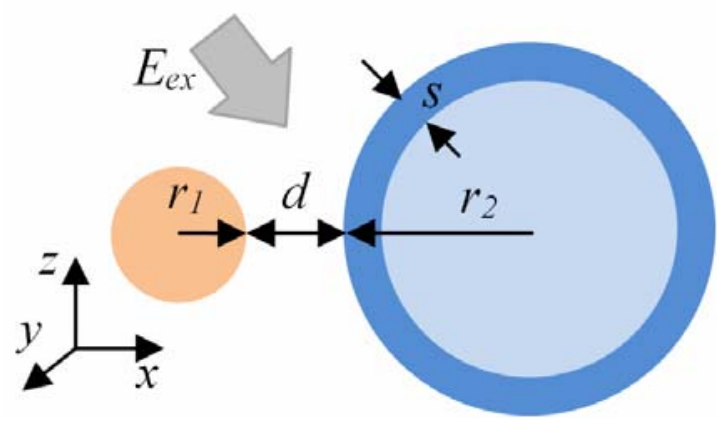

Fig. 1. Sketch of the system.
To calculate the nanoparticle response to the external field, the Lippmann-Schwinger equation can be used [15]:

$$
\begin{aligned}
& E_{i}(\mathbf{R})=E_{i}^{0}(\mathbf{R})+ \\
& +k_{0}^{2} \int_{V} d \mathbf{R}^{\prime} G_{i j}\left(\mathbf{R}, \mathbf{R}^{\prime}\right) \chi_{j k}\left(\mathbf{R}^{\prime}\right) E_{k}\left(\mathbf{R}^{\prime}\right),
\end{aligned}
$$

where $E_{i}(\mathbf{R})$ is the self-consistent local field, $E_{i}^{0}(\mathbf{R})-$ external field, $k_{0}$ - wave number, $G_{i j}\left(\mathbf{R}, \mathbf{R}^{\prime}\right)$ - photon propagator, $\chi_{j k}(\mathbf{R})$ - susceptibility of the nanoparticle.

This integral equation can be solved using the concept of effective susceptibility [16]:

$$
\begin{aligned}
& E_{i}(\mathbf{R})=E_{i}^{0}(\mathbf{R})+ \\
& +k_{0}^{2} \int_{V} d \mathbf{R}^{\prime} G_{i j}\left(\mathbf{R}, \mathbf{R}^{\prime}\right) \mathrm{X}_{j k}\left(\mathbf{R}^{\prime}\right) E_{k}^{0}\left(\mathbf{R}^{\prime}\right), \\
& \mathrm{X}_{i j}(\mathbf{R})=\chi_{i k}(\mathbf{R})\left(\delta_{k j}-S_{k j}(\mathbf{R})\right)^{-1} \\
& S_{k j}(\mathbf{R})=k_{0}^{2} \int_{V} d \mathbf{R}^{\prime} G_{k i}\left(\mathbf{R}, \mathbf{R}^{\prime}\right) \chi_{i j}\left(\mathbf{R}^{\prime}\right),
\end{aligned}
$$

Where $\mathrm{X}_{i j}(\mathbf{R})$ is the effective susceptibility of the nanoparticle. For the system of two objects interacting with each other, Eqs (2) and (4) can be written as:

$$
\begin{aligned}
& E_{i}(\mathbf{R})=E_{i}^{0}(\mathbf{R})+ \\
& +k_{0}^{2} \int_{V_{1}} d \mathbf{R}^{\prime} G_{i j}\left(\mathbf{R}, \mathbf{R}^{\prime}\right) \mathrm{X}_{j k}^{(1)}\left(\mathbf{R}^{\prime}\right) E_{k}^{0}\left(\mathbf{R}^{\prime}\right)+ \\
& +k_{0}^{2} \int_{V_{2}} d \mathbf{R}^{\prime} G_{i j}\left(\mathbf{R}, \mathbf{R}^{\prime}\right) \mathrm{X}_{j k}^{(2)}\left(\mathbf{R}^{\prime}\right) E_{k}^{0}\left(\mathbf{R}^{\prime}\right) \\
& \mathrm{X}_{i j}^{(1,2)}(\mathbf{R})=\chi_{i k}^{(1,2)}(\mathbf{R})\left(\delta_{k j}-S_{k j}(\mathbf{R})\right)^{-1} \\
& S_{k j}(\mathbf{R})=k_{0}^{2} \int_{V_{1}} d \mathbf{R}^{\prime} G_{i j}\left(\mathbf{R}, \mathbf{R}^{\prime}\right) \chi_{j k}^{(1)}\left(\mathbf{R}^{\prime}\right)+ \\
& +k_{0}^{2} \int_{V_{2}} d \mathbf{R}^{\prime} G_{i j}\left(\mathbf{R}, \mathbf{R}^{\prime}\right) \chi_{j k}^{(2)}\left(\mathbf{R}^{\prime}\right) .
\end{aligned}
$$$$
\text { Here } \mathrm{X}_{j k}^{(1)}, \mathrm{X}_{j k}^{(2)} \text { are effective susceptibilities of }
$$

first and second object respectively. It should be noted that the denominator in (6) is the same in both cases. If effective susceptibility functions of each object separately are known, we can write each effective susceptibility function with interaction between them:

$$
\begin{aligned}
& \mathrm{X}_{i j}^{(1,2)}(\mathbf{R})=\chi_{i k}^{(1,2)}(\mathbf{R})\left[\chi_{k l}^{(1,2)}(\mathbf{R})\left(\Xi_{l j}^{(1,2)}(\mathbf{R})\right)^{-1}-\right. \\
& \left.-k_{0}^{2} \int_{V_{1}} d \mathbf{R}^{\prime} G_{k l}\left(\mathbf{R}, \mathbf{R}^{\prime}\right) \chi_{i j}^{(2,1)}\left(\mathbf{R}^{\prime}\right)\right]^{-1},
\end{aligned}
$$

(C) 2016, V. Lashkaryov Institute of Semiconductor Physics, National Academy of Sciences of Ukraine 
where $\Xi_{j k}^{(1,2)}(\mathbf{R})$ is the effective susceptibility of the first and second objects separately from each other. For the spherical nanoparticle, the effective susceptibility can be written as that in [17]:

$$
\mathrm{X}_{i j}(\mathbf{R})=\chi_{i k}(\mathbf{R})\left(\delta_{k j}+\chi_{k j}(\mathbf{R}) / 3\right)^{-1}
$$

The system under consideration represents the spherical nanoparticle near the virus irradiated by external field. So, using (8) and (9), the effective susceptibility of virus and nanoparticle can be written as: $\mathrm{X}_{i j}^{V}(\mathbf{R})=\chi_{i k}^{V}(\mathbf{R})\left[\delta_{k j}+\chi_{k j}^{V}(\mathbf{R}) / 3-\right.$

$\left.-k_{0}^{2} \int_{V_{p}} d \mathbf{R}^{\prime} G_{k l}\left(\mathbf{R}, \mathbf{R}^{\prime}\right) \chi_{l j}^{p}\left(\mathbf{R}^{\prime}\right)\right]^{-1}$,

$\mathrm{X}_{i j}^{p}(\mathbf{R})=\chi_{i k}^{p}(\mathbf{R})\left[\delta_{k j}+\chi_{k j}^{p}(\mathbf{R}) / 3-\right.$

$\left.-k_{0}^{2} \int_{V_{V}} d \mathbf{R}^{\prime} G_{k l}\left(\mathbf{R}, \mathbf{R}^{\prime}\right) \chi_{l j}^{V}\left(\mathbf{R}^{\prime}\right)\right]^{-1}$,

where $\chi_{i j}^{V}(\mathbf{R})$ and $\chi_{i j}^{p}(\mathbf{R})$ are the susceptibilities of virus and nanoparticle, respectively; $V_{p}, V_{V}$ - their volumes. Using (5) and (10), (11), local field distributions can be easily calculated. It should be noted that the denominator of Eqs. (10), (11) can be equal to zero, which means the effective susceptibility and the local field (2) can increase very much. Of course, in real system only the real part of denominator will be equal to zero, but imaginary part will not be. In conclusion, that is the reason of local field enhancement effect in the system, and it depends on position of the objects.

For numerical calculation, the following system parameters were used: the radius of the nanoparticle $6 \mathrm{~nm}$; dielectric permittivity of the nanoparticle is $-10.5+1.3 i$ (gold); radius of the virus $-60 \mathrm{~nm}$; the shell thickness $-10 \mathrm{~nm}$; dielectric permittivity of the inner part of the virus -1.5 ; dielectric permittivity of the shell -4.5 ; wavelength of the external field $632 \mathrm{~nm}$; distance between the virus and nanoparticle $1 \mathrm{~nm}$. External field propagates along X-axis with Zpolarization. Results of calculations are shown in Fig. 2 .

As we can see, there is a local field enhancement in the shell of the virus. It can be the reason of decrease in the virus activity. It is noteworthy that with another field direction there is no enhancement at all, but the real system permanently flows and in some time moment we will obtain a considered setup of the system.

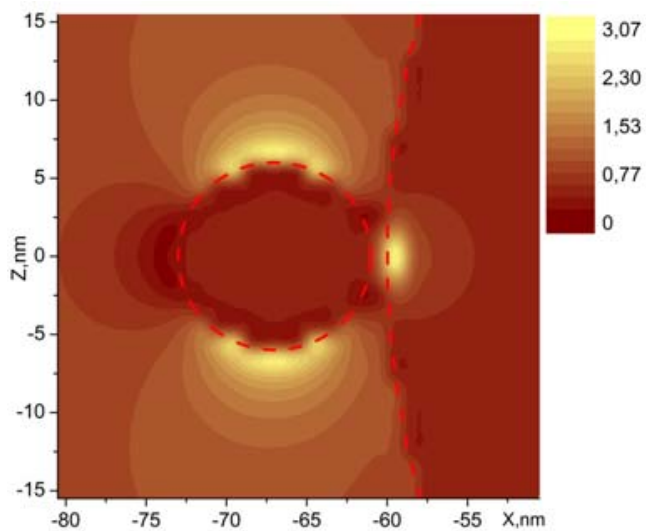

Fig. 2. Local field intensity distribution near the gold nanoparticle and the shell of the virus.

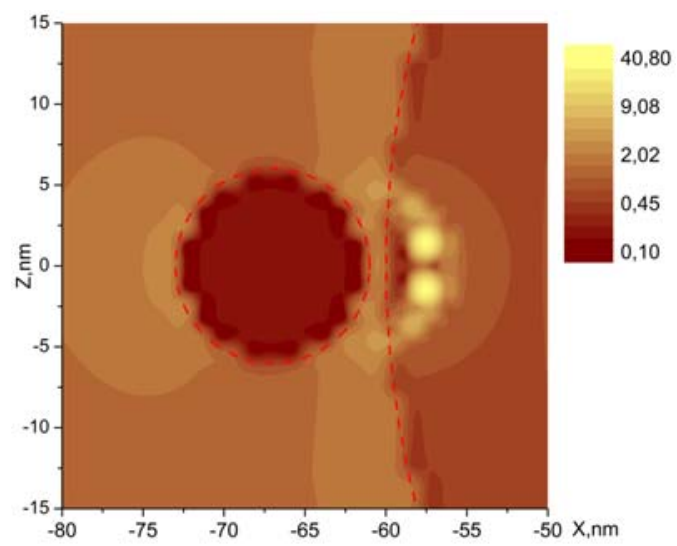

Fig. 3. Local field intensity distribution near the silicon nanoparticle and the shell of the virus.

For another calculation (Fig. 3), the silicon nanoparticle with the dielectric permittivity $-15.05+0.008 i$ and external field propagating along Zaxis with $\mathrm{X}$-polarization were chosen, because of the same reason - with another field direction there is no enhancement.

In that case, local field enhancement is much greater than with gold nanoparticle. It happens because the imaginary part of dielectric permittivity for silicon is much lower than that of gold. We supposed that dielectric and semiconductor nanoparticles can be effective in antiviral therapy and may be better antiviral agents than the metal ones.

\section{Absorption spectra of the "virus+nanoparticles" preparations}

It is well known that the absorption spectrum presents information about composition of the sample. Thus, it is natural to suppose that interaction between a virus and nanoparticle influences on absorption of the sample and could be studied by measuring and analyzing its absorption spectrum. 
Table 1. Bands parameters of vesicular stomatitis virus samples with and without nanoparticles, obtained from the absorption spectra in the visible range.

\begin{tabular}{|c|c|c|c|c|c|c|c|c|}
\hline \multirow{2}{*}{$i$} & \multicolumn{2}{|c|}{$\lambda_{i}, \mathrm{~nm}$} & \multicolumn{2}{c|}{$E_{i}, \mathrm{eV}$} & \multicolumn{2}{c|}{$\gamma_{i}, \mathrm{eV}$} & \multicolumn{2}{c|}{$f_{i}, \mathrm{eV}^{2}$} \\
\cline { 2 - 10 } & Without np & $\begin{array}{c}\text { With } \\
\mathrm{np}\end{array}$ & Without np & With np & Without np & With np & Without np & With np \\
\hline 1 & 468 & 468 & 4.777 & 4.777 & 0.318 & 0.318 & 0.2 & 0.19 \\
\hline 2 & 541 & 541 & 4.358 & 4.358 & 0.318 & 0.318 & 1.54 & 1.51 \\
\hline 3 & 644 & 640 & 4.21 & 4.24 & 0.318 & 0.483 & 2.23 & 1.98 \\
\hline
\end{tabular}

Table 2. Third oscillator parameters of vesicular stomatitis virus preparations with and without nanoparticles, obtained from the absorption spectra in the visible range.

\begin{tabular}{|c|c|c|c|c|}
\hline NP concentration & $\lambda_{i}, \mathrm{~nm}$ & $E_{i}, \mathrm{eV}$ & $\gamma_{i}, \mathrm{eV}$ & $f_{i}, \mathrm{eV}^{2}$ \\
\hline 0 & 644 & 4.21 & 0.318 & 2.23 \\
\hline $0.01 \mathrm{nM}$ & 640 & 4.24 & 0.483 & 1.98 \\
\hline $0.1 \mu \mathrm{M}$ & 638 & 4.25 & 0.501 & 1.73 \\
\hline $0.01 \mathrm{mM}$ & 638 & 4.25 & 0.511 & 1.52 \\
\hline
\end{tabular}

For the experiments, we used a sample of vesicular stomatitis viruses, samples of vesicular stomatitis viruses with $\mathrm{CeO}_{2}$ nanoparticles in different concentrations, a buffer, where the viruses were dissolved, and a growth medium, where the viruses have been grown. In the work, the changes of the buffer components concentrations were not taken into account because of their smallness (the biggest concentration value is about $50 \mathrm{ng} / \mathrm{ml}$ ). All the samples were kindly supplied by scientists of the D. Zabolontyi Institute of Microbiology and Virology, NAS of Ukraine, Kyiv, Ukraine (N. Zholobak, O. Shydlovska). The samples were irradiated with halogen lamp through the lens, and transmitted light was collected by a photoelectric multiplier. The gained signal was processed with computer program. Absorption was calculated as a difference between the light intensity transmitted through the cuvette with the buffer and that transmitted through the cuvette with the sample. All the experiments were repeated for five times during one day with the period of one hour in order to eliminate the possibility of virus destruction in the sample due to the effect of light. Absorption spectra were measured for the samples of pure viruses with different virus concentrations, for the samples of viruses with nanoparticles, for the samples of viruses in one week after their preparation and after one-day illumination with direct sunlight, for the samples of buffer and growth medium.

As a result of series of experiments, we obtained the absorption spectra of vesicular stomatitis virus sample with taking into account the absorption of the buffer and growth medium in the visible optical range. The extinction coefficient of the preparation has been obtained using the Lambert-Bouguer law.

In order to analyze the spectra, representation of the extinction coefficient dispersion in the form of combination of a finite number of oscillators was used.
It was ascertained that the absorption spectrum of the pure viral sample consists of three bands in the visible range. When the nanoparticles are added to the preparation, the intensities of two bands decrease a little, while the intensity of the another one decreases drastically. Furthermore, the higher the nanoparticles concentration is, the more is the decrease in the band intensities. The results of calculations are presented in Table 1. These results are in good agreement with the results of biomedical experiments on evaluation of the viral infectivity under the action of the nanoparticle samples.

Here, $\gamma_{i}, f_{i}$ are the damping coefficient and the strength of the $i$-th oscillator, respectively, $E_{i}$ - energy of photon with the angular frequency $\omega_{i}, \omega_{i}-$ natural frequency of the $i$-th oscillator.

As the most significant changes took place in the parameters of the third oscillator, its parameters for different concentrations of nanoparticles were calculated. The results are presented in Table 2.

It should be noted that while studying the adsorption spectra of pure virus samples, when the concentration of virus in the sample decreases, all the oscillator strengths decrease uniformly, thus the shape of the spectrum stay the same and just the area under the curve changes.

Absorption spectra of virus samples were also measured in a week after the samples preparation. During six days the samples were kept in refrigerator at the temperature $5{ }^{\circ} \mathrm{C}$ and for one day the samples were kept at the room temperature under direct sunlight. As vesicular stomatitis virus is very unstable, the virus should be inactivated under direct sunlight. These samples demonstrate negligible absorption in the studied wavelength range. It can indicate accordance of these absorption lines to infective virus.

\section{(C) 2016, V. Lashkaryov Institute of Semiconductor Physics, National Academy of Sciences of Ukraine}




\section{Conclusions}

In the work, it has been shown that deformation (or destruction) of the molecules at the virus surface is the possible universal mechanism of inhibition of virus infectivity by nanoparticles. It is caused by the local field enhancement effect.

Performed calculations have shown that the intensity of local field drastically increases when the nanoparticle occurs near the virus. Furthermore, the degree of this decrease depends on the type of the nanoparticle, which is in a good agreement with experimental results obtained by different research teams. Thus, e.g., there is information on the better antiviral action of $\mathrm{CeO}_{2}$ nanoparticles comparatively to the gold ones [10].

Optical measurements performed in the visible range show that the loss of the virus infectivity is caused by the changes in their structure (or structure of molecular ions in its surface, which is supposed). Furthermore, the type and degree of these changes can give information on the sample content (pure virus or virus with nanoparticles) and its infectivity.

\section{Acknowlegements}

We are grateful to N. Zholobak and O. Shydlovska (D. Zabolontyi Institute of Microbiology and Virology, NAS of Ukraine, Kyiv, Ukraine) for preparation of biological samples, and to V. Lozovski (Institute of High Technologies, Taras Shevchenko National University of Kyiv, Kyiv, Ukraine) for fruitful discussions.

\section{References}

1. J. Judd, M.L. Ho, A. Tiwari et al., Tunable protease-activatable virus nanonodes // ACS Nano, 8(5), p. 4740-4746 (2014).

2. S.D. Perrault and W.M. Shih, Virus-inspired membrane encapsulation of DNA nanostructures to achieve in vivo stability // ACS Nano, 8(5), p. $5132-5140$ (2014).

3. R.W.G. Wyckoff, Multiplication of the T3 bacteriophage against E. coli. // Exp. Biol. Med. 71(1), p. 144-146 (1949).

4. S. Matsuzaki, M. Rashel, J. Uchiyama et al., Bacteriophage therapy: a revitalized therapy against bacterial infectious diseases // J. Infect. Chemotherapy, 11, p. 211-219 (2005).

5. C.E. Mire, J.B. Geisbert, K.N. Agans et al., Durability of a vesicular stomatitis virus-based marburg virus vaccine in nonhuman primates // PLoS One, 9(4), p. e94355 (2014).

6. N.A. Mazurkova, Y.E. Spitsyna, N.V. Shikina, Z.R. Ismagilov, S.N. Zagrebel'nyi, and E.I. Ryabchikova, Interaction of titanium dioxide nanoparticles with influenza virus // Ross. Nanotekhnol. 5(5), p. 417-420 (2010).

7. J.L. Elechiguerra, J.L. Burt, J.R. Morones, A. Camacho-Bragado et al., Interaction of silver nanoparticles with HIV-1 // J. Nanobiotechnology, 3(6), Article No. 6 (2005).

8. H.H. Lara, N.V. Ayala-Nuñez, L. Ixtepan-Turrent, C. Rodriguez-Padilla, Mode of antiviral action of silver nanoparticles against HIV-1 // J. Nanobiotechnology, 8(1), Article No. 6 (2010).

9. R.L. Hu, S.R. Li, F.J. Kong, R.J. Hou, X.L. Guan and F. Guo, Inhibition effect of silver nanoparticles on herpes simplex virus 2 // Genetics and Molecular Research, 13(3), p. $7022-7028$ (2014).

10. V. Lysenko, V. Lozovski, M. Spivak, Nanophysics and antiviral therapy // Ukr. J. Phys. 58(1), p. 77-90 (2013).

11. L. Lu, R.W.-Y. Sun, R. Chen et al., Silver nanoparticles inhibit hepatitis B virus replication // Antiviral Therapy, 13, p. 253-262 (2008).

12. Y. Luo, J. Qiu, Human parvovirus B19: a mechanistic overview of infection and DNA replication // Future Virology, 10(2), p. 155-167 (2015).

13. D. R. Beniac, P. L. Melito, S. L. deVarennes et al., The organization of Ebola virus reveals a capacity for extensive, modular polyploidy // PLOS ONE, 7(1), p. e29608 (2012).

14. D.H. Crowford, Viruses: A Very Short Introduction. Oxford University Press, Oxford, 2011.

15. J.D. Jackson, Classical Electrodynamics. 3-rd ed. John Wiley \& Sons, New York, 1998.

16. V. Lozovski, The effective susceptibility concept in the electrodynamics of nano-systems // J. Comput. and Theor. Nanosci. 7(1), p. 1-17 (2010).

17. A.B. Evlyukhin, S.I. Bozhevolnyi, Surface plasmon polariton scattering by small ellipsoid particles // Surf. Sci. 590(2-3), p. 173-180 (2005). 